\title{
THE RELIABILITY OF GINGIVAL CREVICULAR BLOOD AS A DIAGNOSTIC TOOL FOR DIABETES BY USING GLUCOSE SELF-MONITORING DEVICE IN PATIENTS WITH PERIODONTAL DISEASE WITH AND WITHOUT DIABETES
}

\author{
Farhan Butt, Marium Iqbal*, Saima Hanif*, Mohsin Ghiraj*, Faisal, Mudassir Hussain** \\ Armed Forces Institute of Dentistry/National University of Medical Sciences (NUMS) Rawalpindi Pakistan, *Jinnah Medical and Dental College, Karachi Pakistan, \\ **Karachi Medical and Dental College, Karachi Pakistan
}

\begin{abstract}
Objective: To assess the reliability of gingival crevicular blood as a diagnostic tool for diabetes in patients with periodontal disease with and without diabetes.

Study Design: Cross sectional study.

Place and Duration of Study: Department of Periodontics, Jinnah Medical and Dental College, Karachi, Pakistan, from Jul 2017 to Jul 2018.

Methodology: Forty patients with diabetes and 60 patients without diabetes with mild to moderate gingivitis or periodontitis in either the upper or lower anterior region were included. Gingival crevicular blood (GCB) was collected and was assessed by glucometer. The same patient underwent finger stick blood (FSB) and intravenous blood glucose level (IV). Plaque Index (PI), Periodontal Pocket Depth (PPD) and Gingival index were also recorded.

Results: A positive correlation (r) was detected between glucose levels of Gingival crevicular blood with finger stick blood with the value of coefficient correlation ' $r$ ' $=0.849$. The mean values of Gingival index in patients without diabetes is $1.53 \pm$ $0.97 \mathrm{~mm}$, patients with newly diagnosed diabetes is $1.87 \pm 0.920 \mathrm{~mm}$ and without diabetes is $2.13 \pm 0.94 \mathrm{~mm}$.

Conclusion: Blood glucose level can be assessed with the help of Gingival crevicular blood as this technique was found easy and non-invasive to the patient and it can help in diagnosing diabetes during regular periodontal treatment.
\end{abstract}

Keywords: Diabetes mellitus, Finger stick Blood, Glucometer, Periodontitis.

This is an Open Access article distributed under the terms of the Creative Commons Attribution License ( https://creativecommons.org/licenses/by-nc/4.0/), which permits unrestricted use, distribution, and reproduction in any medium, provided the original work is properly cited.

\section{INTRODUCTION}

Chronic periodontitis and diabetes mellitus (DM) are common chronic diseases in adults throughout the world $^{1}$. DM is expected to rise to 333 million in 2025 , as per World Health Organization estimates ${ }^{2}$. It is predicted that $70 \%$ of those affected will be from the developing world ${ }^{2}$. Around 90\% Diabetes Mellitus patients suffer type 2 Diabetes Mellitus i.e. is non-insulin dependent type, and is supposed to be increased up to $6 \%$ annually (Reference by Rees TD) ${ }^{2}$. It is more commonly found in urban population when compared to rural population ${ }^{2}$.

DM is associated with a number of complications, such as altered wound healing, vascular diseases, nephropathy and periodontitis ${ }^{3}$. As per American Diabetes Association in 1993, periodontal disease is the sixth most common complication in patients of Diabetes Mellitus ${ }^{4}$.

Sub-gingival plaque samples taken for microscopic examination from sites with periodontitis has revealed elevated levels of Gram-negative aerobes and Bacteriodes $^{5}$. It is anticipated that in dental setting, for

Correspondence: Dr Saima Hanif, Department of Oral Biology, Jinnah Medical and Dental College, Karachi Pakistan

Received: 25 Sep 2019; revised received: 18 Mar 2020; accepted: 08 May 2020 every known patient with diabetes there is one with undiagnosed $\mathrm{DM}^{6}$. A large number of patients visiting for dental treatment are completely unaware of $\mathrm{DM}^{7}$.

Therefore, the objective of this study was to assess the reliability of gingival crevicular blood as a diagnostic tool for diabetes by using glucose self-monitoring device in patients with periodontal disease with and without diabetes. GCB can be an alternate method to monitor blood glucose level without pain and discomfort to the patient.

\section{METHODOLOGY}

The study was approved by the ethics committee of Jinnah Medical and Dental College Protocol \#: 00008/20. Written informed consent was taken from all the patients prior to start of the procedure. Patient confidentiality was respected. The study population consist of patients visiting the department of Periodontics, Jinnah Medical and Dental College, Karachi, Pakistan from July 2017 to July 2018. It was a cross sectional study which was performed on a total of 100 patients, with diabetes and without diabetes but both with mild or moderate form of chronic periodontitis with an age between 20-65 years. The patients were further divided into three different groups i.e. group 1 (without diabetes), group 2 (undiagnosed diabetes patients) and 
Group 3 (already diagnosed), with mild and moderate periodontitis. Group 2 comprised of those subjects who were initially thought to be without diabetes but were diagnosed with high blood sugar levels during their periodontal examination. Patients that were excluded from the study were those who required prophylactic antibiotic and had hematologic disorder accompanied by an abnormally low or high hematocrit like Polycythemia Vera, anaemia or ones who were taking medicines that interfered with the coagulation system for example, Non-steroidal anti-inflammatory drugs or Heparin. Patients who were on dialysis and those with severe cardio-vascular, hepatic, immunologic, renal, hematological, or other organ disorders were not included.

Patients which were included in the study were between 20-65 years of age and were with or without diabetes but with gingivitis or periodontitis in maxillary or mandibular anterior region.

Samples of gingival crevicular blood were obtained from both patients with and without diabetes. Intraoral clinical examination of each patient was done for signs of gingival inflammation. Bleeding site was selected from mandibular or maxillary anterior teeth, as it is easier to obtain blood from anterior region. One site with bleeding on probing was randomly selected for each measurement. With the help of air drying and gauze, every attempt was made to prevent contamination with saliva. To facilitate collection of blood, in some cases, piece of supra gingival calculus was removed with the ultrasonic scaler. The interdental area in the anterior teeth was probed with UNC-15 (University of North Carolina) probe. Bleeding was observed, as soon as the probe was removed from the gingival sulcus. The test end of the strip, already mounted onto the Glucometer, was brought to the bleeding site to collect blood sample without contamination. The test strip was held in position until the sound of beep was heard from the instrument which gave the gingival crevicular blood glucose reading in $\mathrm{mg} / \mathrm{dl}$.

For finger stick blood (FSB) glucose level, soft cutaneous tissue of middle finger was selected. The soft tissue was first wiped with spirit and was then allowed to evaporate, to prevent contamination. With the help of sterile lancet, the soft tissue surface of the finger was pricked. The test strip was placed near the finger from where the blood was oozing. The instrument was kept in position until the beep was heard and the instrument gave the blood glucose reading in $\mathrm{mg} / \mathrm{dl}$ on its screen.
For the collection of intravenous blood, samples were drawn from the patient's ante-cubital fossa. The surface was wiped with the spirit and it was allowed to evaporate. With the help of disposable syringe, 0.5 $\mathrm{ml}$ of venous blood was collected. This sample of blood was then transferred into the test tube and was immediately sent to laboratory for analysis of blood glucose level. The data all three blood glucose levels were analyzed using SPSS-21. Plaque index (PI Silness and Loe 1964), periodontal pocket depth (PPD) and gingival index (Loe and Silness 1963) was also recorded. Informed consent was taken from the patient before procedure.

\section{RESULTS}

The GCB glucose level in 100 subjects showed that among the subjects with unknown glycemic status, 16 subjects were with diabetes (new patients). Therefore, the study comprised 56 patients with diabetes and 44 patients without diabetes (table-I).

The mean PI and GI in newly diagnosed patients with diabetes was $1.95 \pm 0.951$ and $1.87 \pm 0.920$, in patients without diabetes it was $1.56 \pm 0.941$ and $1.53 \pm$ 0.97 . However it found to be higher among patients with already diagnosed diabetes, which was $2.31 \pm$ 0.607 and $2.13 \pm 0.94$.

Table-I: Mean and standard deviation of all three groups.

\begin{tabular}{l|c}
\hline Variable & Mean \pm SD (mg/dl) \\
\hline Without Diabetes Group & $94.58 \pm 18.66$ \\
Gingival Crevicular Blood & $95.88 \pm 12.95$ \\
Finger Stick Blood & $93.77 \pm 11.08$ \\
Intra Venous & $177.5 \pm 37.51$ \\
\hline \multicolumn{2}{c}{ Newly Diagnosed Diabetes Group } \\
\hline \multicolumn{2}{c}{ Gingival Crevicular Blood } \\
Finger Stick Blood & $184.31 \pm 32.07$ \\
Intra Venous & $184.0 \pm 36.66$ \\
\hline Already Diagnosed Diabetes & $175.05 \pm 63.34$ \\
Gingival Crevicular Blood & $173.40 \pm 65.44$ \\
Finger Stick Blood & $171.43 \pm 65.1$ \\
Intra Venous
\end{tabular}

Table-II: Showing gingival index $(\mathrm{mm})$ and probing pocket depth (mm).

\begin{tabular}{l|c|c}
\hline Group & n & Mean \pm SD \\
\hline Gingival Index & 44 & $1.53 \pm 0.97$ \\
Non Diabetic & 16 & $1.87 \pm 0.920$ \\
Newly Diagnosed & 40 & $2.13 \pm 0.94$ \\
Already Diagnosed & \multicolumn{2}{|c}{$2.76 \pm 0.94$} \\
Probing Pocket Depth & $3.01 \pm 0.91$ \\
Non Diabetic & $3.81 \pm 0.96$. \\
Already Diagnosed &
\end{tabular}




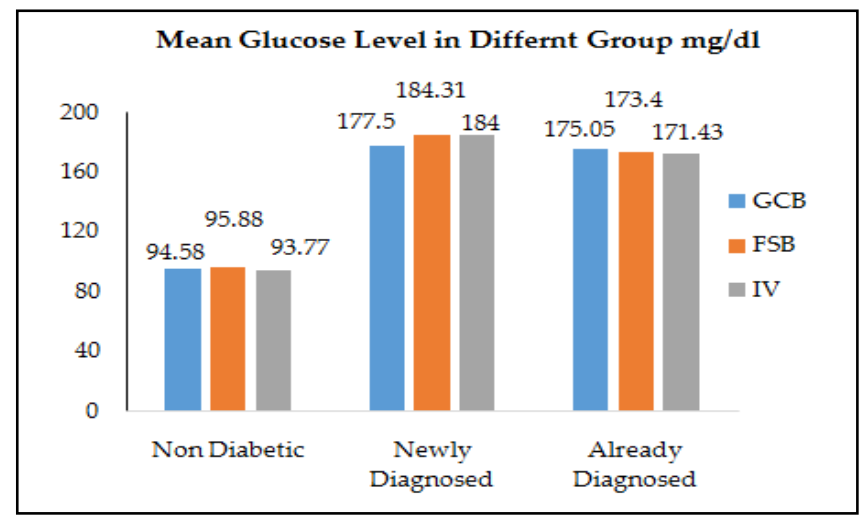

Figure: Difference in glucose level between Gingival crevicular blood, Finger stick blood and Intravenous blood.

\section{DISCUSSION}

DM and periodontitis have shown a two-way association $^{8}$. Uncontrolled DM puts the patient at risk for developing periodontitis further complicates treatment of the disease ${ }^{9}$. This relationship between diabetes and periodontitis can predict that the dental practitioners are likely to encounter a number of undiagnosed diabetes patients with periodontitis ${ }^{10}$. Diabetes mellitus commonly presents with no symptoms in its initial phase and can stay without being diagnosed for many years ${ }^{11}$. Therefore, it is essential to promote opportunities for early diabetes detection, those patients should be especially screened who may be at a high risk for diabetes. Diagnosing type $2 \mathrm{DM}$ cases earlier will help to intervene earlier in the disease course ${ }^{12}$.

Efforts are being made in recent time with regard to the pain free and non-invasive methods to measure blood glucose but only conventional methods are being used for this purpose up till now ${ }^{13}$. Probing during periodontal examination is a routine procedure for a dental practitioner ${ }^{14}$. It is also less traumatic for the patient when compared with finger prick by means of pointed lancet. Intra-oral blood sampling method, can make such tests even more suitable for use by dental practitioners ${ }^{15}$. Gingival crevicular blood by probing, can be a very good source of blood glucose analysis using portable glucose monitor called Glucometer which only needs $3 \mu$ of blood ${ }^{16}$.

Periodontitis is known to produce bleeding during diagnostic periodontal examination which can be used for the routine random blood glucose level estimation ${ }^{17}$. Strauss et al ${ }^{18}$ also reported that GCB samples were suitable in persons with bleeding on probing, to collect a sample without disturbing the tooth or the gingival margin (i.e., in patients having clinical signs of gingivitis or periodontitis). This procedure is fairly simple and non-invasive for the patient than to collect finger stick or IV blood sample. In the present study, IV blood and GCB was used for blood glucose estimation in all 100 subjects. Out of 100 subjects, 16 subjects were diagnosed as new patients with diabetes. These patients were then referred to a physician and he confirmed the results with fasting blood sugar (FBS) and glycosylated hemoglobin estimation.

Current study supported the results of previous researches ${ }^{19}$ as the estimation of glucose level by GCB showed that for the diabetic group, the blood glucose levels were in the range of $175.05 \mathrm{mg} / \mathrm{dl}$, with a mean of $63.34 \mathrm{mg} / \mathrm{dl}$ and for the non-diabetic group, blood glucose levels were in the range of $93.77 \mathrm{mg} / \mathrm{dl}$, with mean of $11.086 \mathrm{mg} / \mathrm{dl}$. The comparison of blood glucose levels of GCB with FSB in our study showed that the $p$-value was $<0.05$ in the groups for GCB with FSB. The correlation coefficient $(\mathrm{r})$ in both the groups showed a positive correlation between glucose levels of GCB with FSB and with the value of 0.84 . In the present study, a glucometer, which is self-timed requires only $1 \mathrm{ml}$ of blood, and requires test strips, which have a capability of inherent capillary action for drawing blood into the test area which is why the collection of blood is easier on the test strip. It was seen that between the two values, the GCB glucose values were reduced than both FSB values in both patients with or without diabetes. This can be due to contamination of GCB by gingival crevicular fluid which probably diluted the glucose concentration producing decreased levels ${ }^{20}$. The significant difference in means of GI and PI of patients with or without diabetes, in our study could be because of etiology of periodontal disease and nature of diabetes. Though, there are patients who suffer from complications after a short period of diabetes, even with a reasonable metabolic control, there are some patients who never encounter periodontal complications even with long standing poorly controlled disease and poor oral hygiene. This is probably due to a variation in individual susceptibility assumed to be related to differences in genetic background ${ }^{21}$. Dentists find intraoral blood glucose sampling technique easier for the diagnosis of DM, as the sample can be obtained through routine scaling and the strip system provides a more convenient method for referral to physicians in comparison to traditionally used medical history review and observation of symptoms.

\section{CONCLUSION}

In conclusion, GCB collected during routine periodontal examination can be a beneficial source of 
blood for analysis by glucometer. The technique was found convenient, less-invasive and less alarming for the patients and therefore, facilitates to the diagnosis of the potential patients with diabetes during periodontal treatment. Dentists hold an important position as a member of the health team and can participate actively in the diagnosis for undiagnosed and asymptomatic DM patients.

\section{CONFLICT OF INTEREST}

This study has no conflict of interest to be declared by any author.

\section{REFERENCES}

1. Patil VS, Patil VP, Gokhale N, Acharya A, Kangokar P. Chronic periodontitis in type 2 diabetes mellitus: oxidative stress as a common factor in periodontal tissue injury. J Clin Diagn Res 2016; 10(4): BC12.

2. Jali MV, Kambar S, Jali SM, Gowda S. Familial early onset of type-2 diabetes mellitus and its complications. North Am J Med Sci 2009; 1(7): 377.

3. International Expert Committee. International Expert Committee report on the role of the A1C assay in the diagnosis of diabetes. Diabetes Care 2009; 32(7): 1327-34.

4. Saremi A, Nelson RG, Tulloch-Reid M, Hanson RL, Sievers ML, Taylor GW, et al. Periodontal disease and mortality in type 2 diabetes. Diabetes Care 2005; 28(1): 27-32.

5. Stewart JE, Wager KA, Friedlander AH, Zadeh HH. The effect of periodontal treatment on glycemic control in patients with type 2 diabetes mellitus. J Clin Periodont 2001; 28(4): 306-10.

6. American Diabetes Association. 2 Classification and diagnosis of diabetes: standards of medical care in diabetes -2018. Diabetes care 2018; 41(Suppl-1): S13-27.

7. American Diabetes Association. Diagnosis and classification of diabetes mellitus. Diabetes care 2014; 37(Suppl-1): S81-90.

8. Nagpal R, Yamashiro Y, Izumi Y. The two-way association of periodontal infection with systemic disorders: an overview. Mediators Inflammation 2015; 2015.
9. Jepsen K, Jepsen S. Antibiotics/antimicrobials: systemic and local administration in the therapy of mild to moderately advanced periodontitis. J Periodontol 2000; 71(1): 82-112.

10. Colombo NH, Shirakashi DJ, Chiba FY, Coutinho MS, Ervolino E, Garbin CAS, et al. Periodontal disease decreases insulin sensitivity and insulin signaling. J Periodontol 2012; 83(7): 864-70.

11. Shetty N, Shankarapillai R, Mathur LK, Manohar B, Mathur A, Jain M. Gingival crevicular blood: As a non-invasive screening tool for diabetes mellitus in dental clinics. J Indian Soc Periodontol 2013; 17(4): 472.

12. Beikler T, Kuczek A, Petersilka G, Flemmig TF. In-Dental-office screening for diabetes mellitus using gingival crevicular blood. J Clin Periodontol 2002; 29(1): 216-18.

13. Turner AP. Biosensors: sense and sensibility. Chem Soc Rev 2013; 42(8): 3184-96.

14. Kaur H, Singh B, Sharma A. Assessment of blood glucose using gingival crevicular blood in diabetic and non-diabetic patients: a chair side method. J Clin Diagn Res 2013; 7(12): 3066.

15. Neville BW, Damm DD, Chi AC, Allen CM. Oral and maxillofacial pathology. Elsevier Health Sciences; 2015.

16. Yadav J, Rani A, Singh V, Murari BM. Prospects and limitations of non-invasive blood glucose monitoring using near-infrared spectroscopy. Biomed Signal Process 2015; 18(1): 214-27.

17. Shetty N, Shankarapillai R, Mathur LK, Manohar B, Mathur A, Jain M. Gingival crevicular blood: As a non-invasive screening tool for diabetes mellitus in dental clinics. J Indian Soc Periodontol 2013; 17(4): 472.

18. Strauss SM, Wheeler AJ, Russell SL, Brodsky A, Davidson RM, Gluzman R, et al. The potential use of gingival crevicular blood for measuring glucose to screen for diabetes: an examination based on characteristics of the blood collection site. J Periodontol 2009; 80(6): 907-14.

19. Parihar S, Tripathi R, Parihar AV, Samadi FM, Chandra A, Bhavsar N. Estimation of gingival crevicular blood glucose level for the screening of diabetes mellitus: A simple yet reliable method. J Oral Biol Craniofacial Res 2016; 6(3): 198-203.

20. Ghimenti S. Chemical information from human fluids for therapy monitoring and clinical diagnosis 2012.

21. American Diabetes Association. Standards of medical care in diabetes-2013. Diabetes Care 2013; 36(Suppl-1): S11. 